\title{
Tumor híbrido parotídeo
}

\author{
Parotid hybrid tumor
}

\author{
Gustavo Bravo $\mathbf{C}^{1}$, Camila Seymour $\mathbf{M}^{2}$, Lara Fernández $\mathbf{R}^{2}$, María Elena Villanueva ${ }^{3}$, Carlos Stott $\mathbf{C}^{1}$, \\ Carlos Celedón L'.
}

\begin{abstract}
RESUMEN
Los tumores de glándulas salivales representan el 3\%-10\% de las neoplasias de cabeza y cuello. La localización más común es en la glándula parótida, representando el $50 \%-85 \%$ de los casos, siendo 20\%-30\% de ellos malignos. Los siguientes son indicadores de malignidad: Crecimiento acelerado, masa dolorosa, parálisis facial asociada y linfoadenopatía. La mayoría de las neoplasias de parótida derivan de un único tipo histológico, pero eventualmente puede ocurrir el desarrollo de más de un tipo en la misma glándula. Este trabajo presenta un caso de una neoplasia en parótida con dos tipos histológicos diferentes, con una presentación clínica atípica.

El paciente se presentó inicialmente con otalgia y otorrea, al examen destacaba un tumor en el canal auditorio externo. El estudio complementario evidenció una neoplasia de parótida y se realizó resección total de la glándula. La biopsia informó un carcinoma adenoideo-quístico con áreas basaloideas diferenciadas. Se administró radioquimioterapia adyuvante, y el control imagenológico con PET-TC mostró la ausencia de recurrencias o diseminación del tumor.

Palabras clave: Cáncer parótida híbrido, tumor híbrido glándula salival.
\end{abstract}

\begin{abstract}
Tumors of the salivary glands represent $33 \%-10 \%$ of head and neck neoplasms. The most common location is the parotid gland, accounting for 50\%-85\% of the cases, with 20\%-30\% of them being malignant. The following are known to be indicative of a malignant tumor: fast growing, painless mass, associated facial paralysis and lymphadenopathy. Most parotid neoplasm derive from a single histological type but eventually the development of more than one type on the same gland can occur. This paper presents a case of a parotid neoplasm with two different histological tumors, with uncharacteristic clinical presentation.

The patient presented initially with ear pain and otorrhoea, in the clinical examination highlighted an external auditory canal tumor. The complementary study revealed a parotid neoplasm and a total resection of the gland was performed. The biopsy revealed an adenoid-cystic carcinoma with differentiated basaloid areas. Adjuvant radio-chemotherapy was administered, and the imaging control with PET-CT showed no evidence of recurrence or dissemination of the tumor.
\end{abstract}

Key words: Parotid hybrid cancer, hybrid salivary gland tumor.

\footnotetext{
Médico. Servicio de Otorrinolaringología, Hospital Clínico Universidad de Chile.

Interna de Medicina, Universidad de Chile.

Médico. Servicio de Anatomía Patológica, Hospital Clínico Universidad de Chile.
} 


\section{INTRODUCCIÓN}

Los tumores de glándulas salivales corresponden al $3 \%$ a $10 \%$ de todos los tumores de cabeza y cuello 1 . Se ha descrito malignidad en $30 \%$ a $40 \%$ de ellos ${ }^{2}$ y, en contraste con la predominancia del carcinoma escamoso en esta localización, en glándulas salivales se han descrito muchos subtipos histológicos, los cuales han sido clasificados en alrededor de 40 tipos por la Organización Mundial de la Salud ${ }^{3}$ (ver Tabla 1). Además, a diferencia de la asociación del tabaco y alcohol a los carcinomas escamosos, la etiología de las neoplasias en glándulas salivales es desconocida. Factores de riesgo aceptados son la exposición a radiación, predisposición genética, tabaco (particularmente en tumor de Warthin), virus y exposición a químicos ambientales ${ }^{4}$.

Estos tumores pueden localizarse tanto en las glándulas salivales mayores (parótida, submandibular y sublingual) como en las menores (distribuidas en la submucosa de la boca y vía aerodigestiva superior) $)^{3,5}$, sin embargo, la mayoría de estas neoplasias se ubican en las glándulas salivales mayores y principalmente en parótida, en la que se encuentra el $50 \%$ al $85 \%$ de los tumores de glándulas salivales ${ }^{6,7}$. En dicha glándula, $20 \%$ a 30\% de los tumores son malignos y, de éstos, los más frecuentes son el carcinoma mucoepidermoide, el carcinoma adenoideo quístico y el adenocarcinoma ${ }^{2,3,6}$. Aproximadamente el $40 \%$ de ellos son indolentes y se presentan como aumento de volumen, sin embargo los indicadores de malignidad más aceptados son su rápido crecimiento, el dolor, el compromiso del nervio facial y la presencia de adenopatía cervical $^{8}$. Las metástasis más frecuentes son en pulmón, seguidas por hueso e hígado ${ }^{9}$. Con respecto a este punto, particularmente el carcinoma adenoideoquístico se asocia a un alto riesgo de metástasis a distancia.

Tabla 1. Clasificación histológica de la OMS de tumores de glándulas salivales

\begin{tabular}{|l|l|}
\hline Tumores epiteliales malignos & Tumores epiteliales benignos \\
\hline Carcinoma células acinares & Adenoma pleomorfo \\
Carcinoma mucoepidermoide & Mioepitelioma \\
Carcinoma adenoideo-quístico & Adenoma células basales \\
Adenocarcinoma polimorfo de bajo grado & Tumor de Warthin \\
Carcinoma epitelial-mioepitelial & Oncocitoma \\
Carcinoma células claras & Adenoma canalicular \\
Adenocarcinoma células basales & Adenoma sebáceo \\
Carcinoma sebáceo & Linfoadenoma \\
Linfoadenocarcinoma sebáceo & Sebáceo \\
Cistoadenocarcinoma & No sebáceo \\
Cistoadenocarcinoma cribiforme de bajo grado & Papiloma ductal \\
Adenocarcinoma mucinoso & Papiloma ductal invertido \\
Carcinoma oncocítico & Papiloma intraductal \\
Carcinoma ductos salivales & Sialoadenoma papilifero \\
Adenocarcinoma & Cistoadenoma \\
Carcinoma mioepitelial & Tumores tejidos blandos \\
Carcinoma ex adenoma pleomorfo & Hemangioma \\
\cline { 2 - 2 } Carcinosarcoma & Tumores hematolinfáticos \\
Metástasis adenoma pleomorfo & Linfoma de Hodgkin \\
Carcinoma células escamosas & Linfoma difuso de células B grandes \\
Carcinoma células pequeñas & Linfoma extranodal de zona marginal de células B \\
Carcinoma células grandes & \\
Carcinoma linfoepitelial & \\
Sialoblastoma & \\
\hline
\end{tabular}

Adaptado de: Barnes L, Eveson JW, Reichart P, Sidransky D. World Health Organization Classification of Tumors. Pathology and Genetics of Head and Neck Tumours, IARC Press, Lyon 2005. (3). 
En un estudio realizado por uno de los autores, se hizo una revisión retrospectiva de su experiencia clínica en tumores de glándulas salivales durante 10 años. Del total de la muestra analizada, 25 casos correspondieron a tumores malignos. En ellos, la edad de promedio fue de 52 años y las manifestaciones más frecuentes, el aumento de volumen y dolor. Elementos como dolor, parálisis facial e histología, representaron factores de mal pronóstico. El $80 \%$ se localizó en parótida, $16 \%$ en glándula submaxilar, y $4 \%$ en glándula salival menor. En cuanto a la histología, hubo predominio de carcinoma mucoepidermoide (28\%) y adenocarcinoma $(16 \%)^{10}$.

La mayoría de las neoplasias en parótida son de un único tipo histológico pero eventualmente se da la ocurrencia de más de un tipo en una misma glándula, siendo ambos malignos o benignos, 0 una combinación de ellos 0 , en otros casos, pueden existir múltiples neoplasias malignas en distintas glándulas ${ }^{4,11,12}$. Un tipo particular de combinación histológica se da en las neoplasias híbridas, en las cuales dos tipos histológicos distintos comparten una misma área geográfica ${ }^{13}$.

El objetivo de este trabajo es presentar un caso clínico en el que se documenta la ocurrencia de una neoplasia híbrida en una glándula salival mayor con presentación clínica no característica.

\section{DESCRIPCIÓN DEL CASO CLÍNICO}

Paciente de sexo masculino de 34 años, sin antecedentes mórbidos, presenta cuadro iniciado en octubre de 2010 caracterizado por otalgia y otorrea fétida en oído derecho. Se indica tratamiento antibiótico vía oral y tópico por diez días, sin embargo, cinco días postratamiento reinicia la misma sintomatología agregándose aumento de volumen en conducto auditivo externo (CAE) derecho, que sangra en forma ocasional, por lo que consulta en el Servicio de Otorrinolaringología del Hospital Clínico de la Universidad de Chile (HCUCH). Se realiza aseo en forma ambulatoria y se toma muestra para estudio anatomopatológico, que informa dermatitis crónica y daño solar actínico. Por persistencia de sintomatología y sospecha de malignidad, se decide la toma de biopsia amplia de la pared anterior del CAE. Se solicita tomografía axial computarizada (TC) de oído con contraste, que informa dos lesiones polipoideas en CAE derecho, la de mayor tamaño más externa asociada a discretos cambios erosivos del conducto óseo, y se sugiere estudio dirigido (ver Figura 1).

En noviembre de 2010 se realiza resección del tumor de CAE y se envía muestra a biopsia, la que informa adenocarcinoma de CAE derecho, de origen apocrino.

Se realiza TC de cuello la que muestra lesión expansiva en espacio parotídeo derecho, de borde parcialmente definido, con leve realce con medio de contraste, en estrecho contacto con CAE y articulación témporo-mandibular derecha. Mide aprox $2 \times 1,9 \times 2,4 \mathrm{~cm}$, en eje transversal, anteroposterior y longitudinal, respectivamente. Existe fenómeno de aspecto erosivo en porción ósea del CAE, en estrecho contacto con masa. No se describen adenopatías y resto de estructuras sin alteraciones (ver Figura 2).

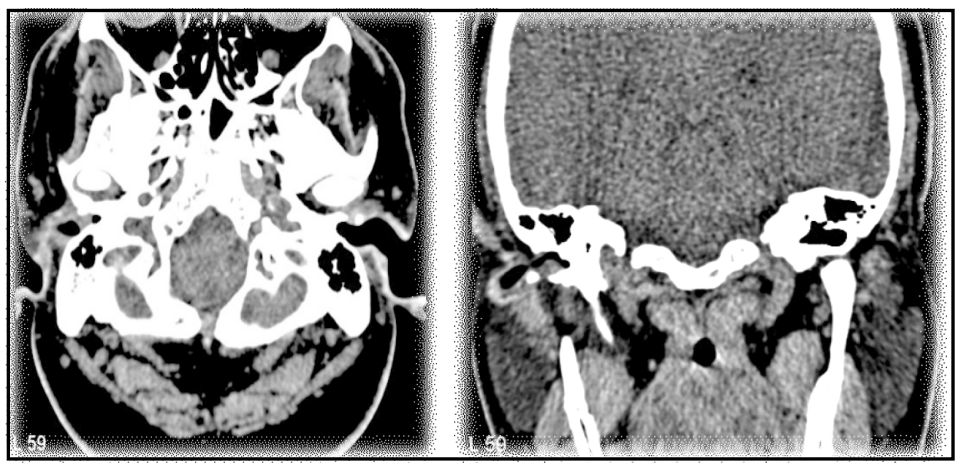

Figura 1. TAC de oídos con contraste preoperatoria. 


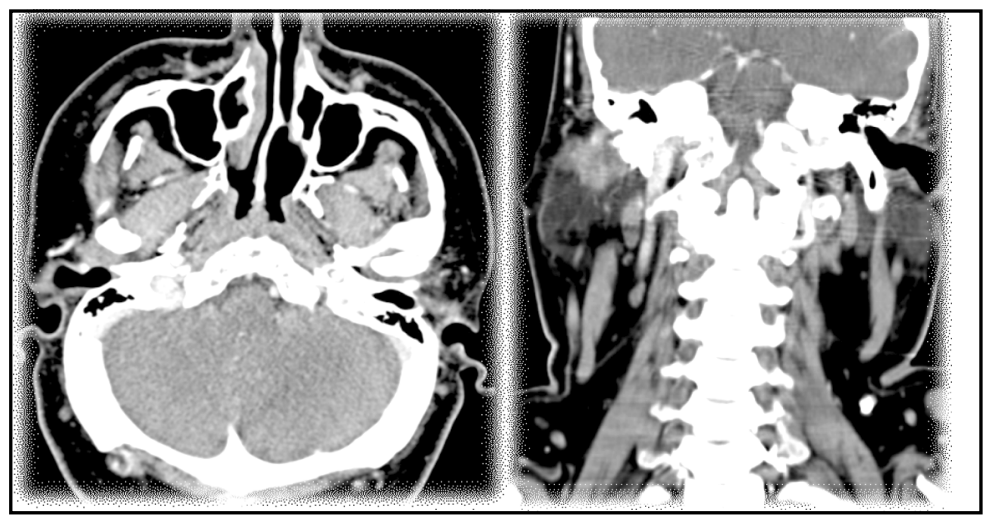

Figura 2. TAC de cuello preoperatoria.

De acuerdo a estudio anatomopatológico e imagenológico, se realiza cirugía a finales de noviembre del mismo año en Hospital Clínico de la Universidad de Chile, correspondiente a una parotidectomía total derecha y resección en bloque del CAE, tanto óseo como cartilaginoso, además de resecar trago y antitrago. Durante la cirugía se identifica nervio facial y se respeta. Biopsia rápida intraoperatoria informa ambos extremos de la muestra (superficial y profundo) sin lesión tumoral. Solución de continuidad a nivel del CAE se cubre con injerto dermo-epidérmico. El informe anatomopatológico informa carcinoma parotídeo con estirpe pendiente por mayor estudio inmunohistoquímico, de 2,3×1 cm, con límites quirúrgicos libres de lesión, con invasión vascular linfática y venosa masiva, sin invasión neural ni compromiso ganglionar en tres ganglios intraparotídeos. Pieza quirúrgica se muestra en Figura 3.

Paciente evoluciona en posoperatorio inmediato en buenas condiciones generales, hemodinámicamente estable, con adecuada apertura bucal y paresia de rama mandibular facial derecha, siendo dado de alta al quinto día posoperatorio, previo retiro de drenaje. En siguientes controles posoperatorios se constata buena evolución, con curaciones seriadas y uso de terapia antibiótica. Se rescata biopsia definitiva que informa carcinoma con áreas de diferenciación basaloide y áreas adenoídeo-quísticas. El estudio inmunohistoquímico fue positivo para S-100, actina y C-kit. Se discute caso en Comité de Cabeza y Cuello del HCUCH, se etapifica el tumor en estadio IVA (T4a, NO, MO) y se acuerda completar tratamiento con quimiorradioterapia adyuvante. Muestras de anatomía patológica de pieza quirúrgica se muestran en Figura 4.

Entre enero y febrero de 2011 se realiza radioterapia de lecho tumoral con irradiación linfonodal electiva, con una dosis de 66Gy, asociado a quimioterapia con cisplatino. La tolerancia al tratamiento combinado fue regular evolucionando con una mucositis y odinofagia, por lo que requirió instalación de

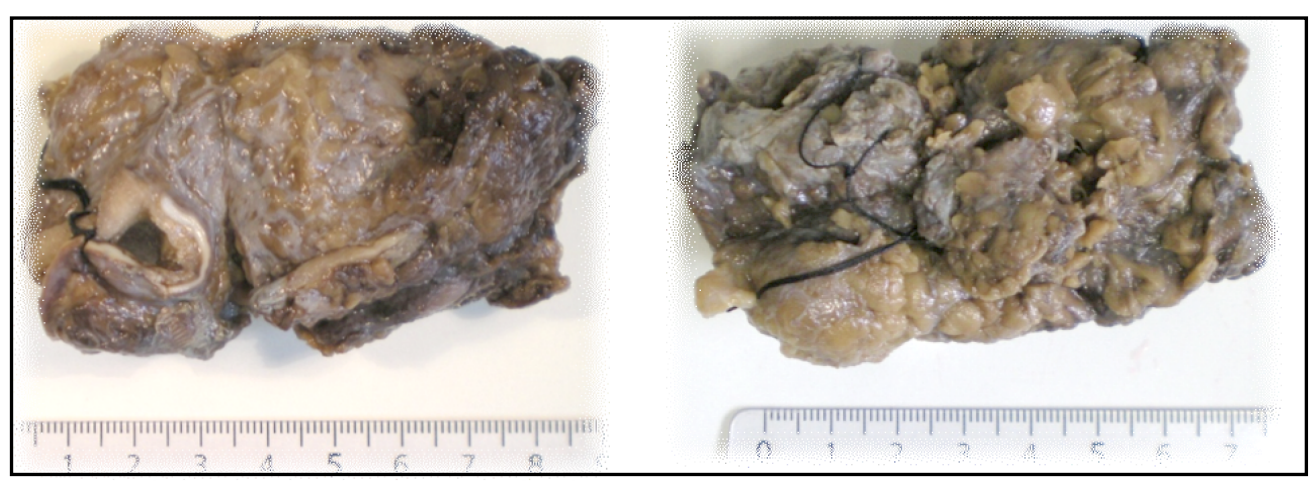

Figura 3. Pieza quirúrgica de la cirugía. 


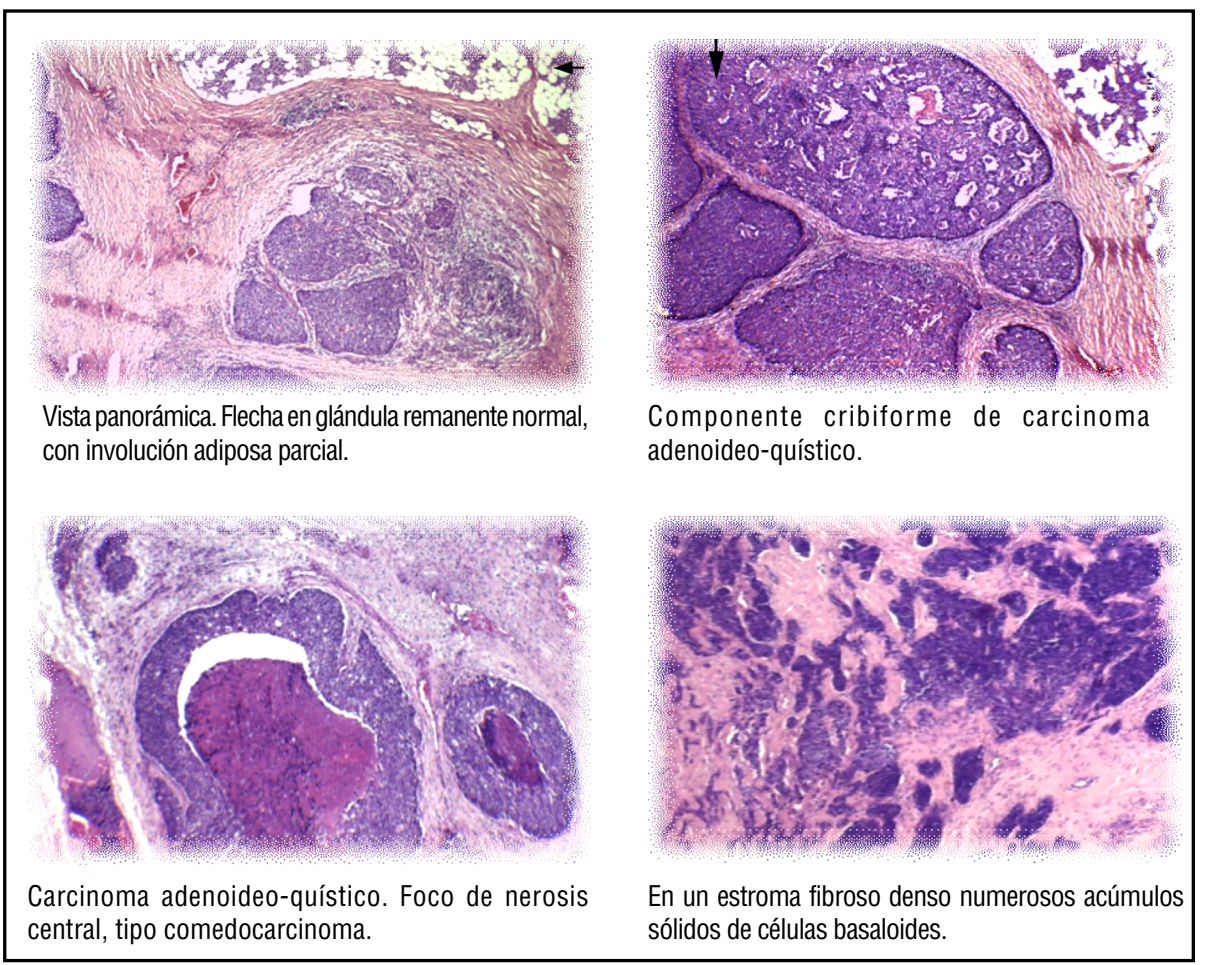

Figura 4. Anatomía patológica de cortes de pieza quirúrgica.

gastrostomía que fue retirada a finales de febrero. El injerto se conservó en buenas condiciones.

En mayo de 2011 se solicita control imagenológico con PET-TC de cuerpo entero, que no muestra evidencia de recidiva local ni diseminación a distancia (ver Figura 5). Resultado estético posquirúrgico se muestra en Figura 6.

\section{DISCUSIÓN}

La parótida concentra la mayoría de los tumores de glándula salivales, y ante un aumento de volumen de la glándula es importante descartar la existencia de una neoplasia, ya sea benigna o maligna. Según la literatura, datos clínicos que indican sospecha de malignidad son un tumor de más de $3 \mathrm{~cm}$, dolor, crecimiento rápido, parálisis facial, infiltración 0 ulceración de la piel y presencia de adenopatías cervicales, sin embargo, la existencia de estos signos también depende del tipo histológico del tumor.
Según Oliva y cols, la mayoría de estas manifestaciones tendrían una baja frecuencia en la presentación de tumores malignos, mientras que el tamaño tumoral mayor a $5 \mathrm{~cm}$ y la edad sobre 46 años serían características clínicas significativas de malignidad $^{14}$. Nuestro caso clínico se presentó con tumoración a nivel del conducto auditivo externo del mismo lado de la neoplasia, la cual se diagnosticó tras un acabado estudio imagenológico e histológico, y no existía aumento de volumen parotídeo.

Al encontrar dos tipos histológicos en una biopsia de una misma glándula salival, se debe tener en cuenta diversas posibilidades como son tumores unilaterales multifocales sincrónicos, carcinomas con cambios metaplásicos, carcinomas desdiferenciados, carcinoma derivado de un adenoma preexistente, tumores híbridos, tumores colisionantes, entre otros. Los tumores híbridos corresponden a una entidad rara, descrita como un tumor compuesto por dos tipos histológicos que ocupan una misma área topográfica ${ }^{15}$. La presencia de una zona transicional 


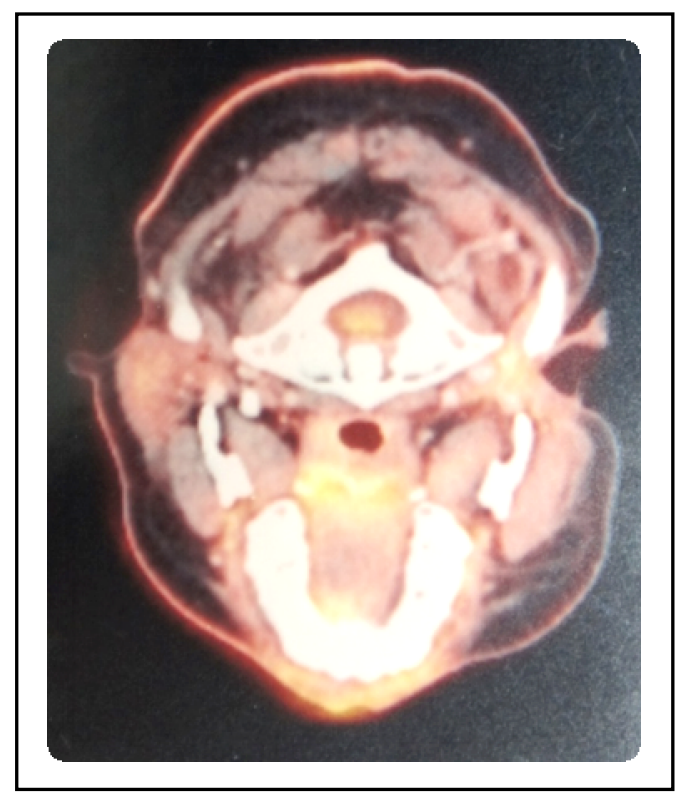

Figura 5. PET-TC posoperatoria.

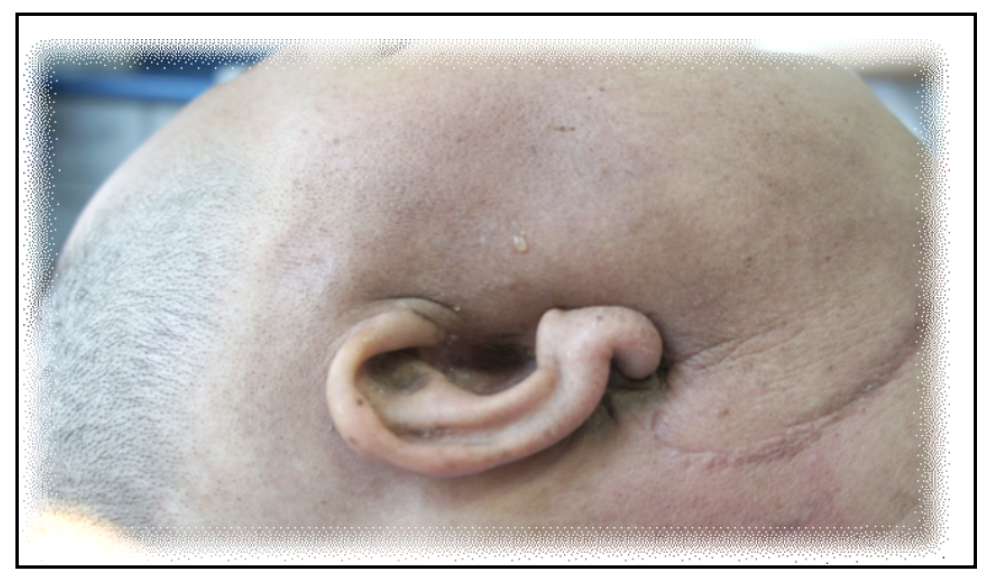

Figura 6. Resultados estéticos posoperatorios.

entre los dos componentes histológicos, que sugiere un mismo origen, diferencia estos tumores de los colisionantes (encuentro de dos carcinomas provenientes de distintas áreas topográficas) y de los múltiples sincrónicos (diversos focos de tumores de igual 0 distinto tipo histológico) ${ }^{13}$.

Los tumores híbridos constituyen el $0,1 \%{ }^{15}$ al $0,4 \%$ de todos los tumores de glándulas salivales ${ }^{16}$ y se han descrito hasta el momento, de nuestro conocimiento, 28 casos en la literatura con diversas combinaciones histológicas, los que se detallan en la Tabla 2. Existe un solo caso descrito compuesto por carcinoma adenoideo quístico y carcinoma basaloide reportado por Ellis ${ }^{17}$.

Para el correcto diagnóstico de los tipos histológicos se ha utilizado el análisis clinicopatológico y la inmunohistoquímica, existiendo algunos reportes controversiales en cuanto 
Tabla 2. Tumores híbridos de glándulas salivales

\begin{tabular}{|c|c|c|c|c|c|c|}
\hline Caso & Edad & Sexo & Glándula salival & Tamaño (cm) & Histología & Autores \\
\hline 1 & - & - & Paladar & 2,5 & EMC/AdCC/BCAc & Ellis et al. 1991 \\
\hline 2 & 70 & M & Parótida & $7 \times 6 \times 4$ & $\mathrm{BCA} / \mathrm{CA}$ & Seifert and Donath 1996 \\
\hline 3 & 62 & M & Parótida & - & BCA/AdCC & Seifert and Donath 1996 \\
\hline 4 & 60 & M & Parótida & $5 \times 2,5 \times 3$ & $\mathrm{AdCC} / \mathrm{WT} / \mathrm{SL}$ & Seifert and Donath 1996 \\
\hline 5 & 53 & M & Parótida & $6 \times 3 \times 2$ & $\mathrm{ACC} / \mathrm{SdC}$ & Seifert and Donath 1996 \\
\hline 6 & 66 & $\mathrm{~F}$ & Paladar & - & EMC/AdCC & Seifert and Donath 1996 \\
\hline 7 & 67 & $\mathrm{~F}$ & Parótida & 5,5 & $\mathrm{ACC} / \mathrm{MC}$ & Ballestin et al.1996 \\
\hline 8 & 51 & M & Paladar & $4,5 \times 3$ & $\mathrm{AdCC} / \mathrm{SdC}$ & Kamio et al. 1997 \\
\hline 9 & 62 & $\mathrm{~F}$ & Parótida & 3 & EMC/AdCC & Simpson 1997 \\
\hline 10 & 53 & M & Parótida & $6 \times 4,5 \times 3,5$ & $\mathrm{AdCC} / \mathrm{MC}$ & Cloitoru et al. 1999 \\
\hline 11 & 71 & M & Parótida & 2,9 & AdCC/EMC & Cloitoru et al. 1999 \\
\hline 12 & 28 & M & Parótida & $2,5 \times 2$ & $\mathrm{EMC} / \mathrm{SdC}$ & Cloitoru et al. 1999 \\
\hline 13 & 51 & M & Paladar & $3,5 \times 3$ & $\mathrm{AdCC} / \mathrm{SdC}$ & Cloitoru et al. 1999 \\
\hline 14 & 36 & $\mathrm{~F}$ & Subman-dibular & $3,5 \times 2,5 \times 2$ & $\mathrm{SdC} / \mathrm{AdCC}$ & Snyder and Paulino 1999 \\
\hline 15 & 78 & $\mathrm{~F}$ & Parótida & $4,5 \times 4 \times 3$ & $\mathrm{PLGA} / \mathrm{SdC} / \mathrm{AdCC} / \mathrm{ACC}$ & Zardawi 2000 \\
\hline 16 & 58 & M & Parótida & 2,5 & EMC/MC & Chetty et al. 2000 \\
\hline 17 & 74 & $\mathrm{~F}$ & Parótida & 10 & $\mathrm{EMC} / \mathrm{BCAC}$ & Toshitaka et al. 2002 \\
\hline 18 & 56 & M & Parótida & 2 & $\mathrm{EMC} / \mathrm{BCAC}$ & Toshitaka et al. 2002 \\
\hline 19 & 73 & $\mathrm{~F}$ & Parótida & 2 & EMC/SCC & Toshitaka et al. 2002 \\
\hline 20 & 40 & M & Parótida & 3 & SdC/AdCC & Toshitaka et al. 2002 \\
\hline 21 & 81 & $\mathrm{~F}$ & Subman-dibular & 3 & SdC/AdCC & Toshitaka et al. 2002 \\
\hline 22 & 65 & M & Parótida & 5 & $\mathrm{MC} / \mathrm{SdC}$ & Toshitaka et al. 2002 \\
\hline 23 & 42 & M & Parótida & 4 & $A C C / S d C$ & Toshitaka et al. 2002 \\
\hline 24 & 56 & M & Parótida & 3,5 & SCC/SdC & Toshitaka et al. 2002 \\
\hline 25 & 49 & $\mathrm{~F}$ & Paladar & $3,5 \times 3,5 \times 2,5$ & MC/AdCC & Ruiz-Godoy et al. 2003 \\
\hline 26 & 71 & M & Paladar & $4 \times 3 \times 3$ & EMC/AdCC & Ruiz-Godoy et al. 2003 \\
\hline 27 & - & $\mathrm{F}$ & Parótida & 4 & LC/EMC & Piana et al. 2004 \\
\hline 28 & 74 & M & Parótida & - & $\mathrm{EMC} / \mathrm{SdC}$ & Kainuma et al. 2010 \\
\hline
\end{tabular}

BCA: Adenoma de células basales; CA: Adenoma canalicular; AdCC: Carcinoma adenoideo-quístico; WT: Tumor de Warthin; SL: Linfoadenoma sebáceo; ACC: Carcinoma de células acinares; SdC: Carcinoma del ducto salival; EMC: Carcinoma epitelialmioepitelial; BCAc: Adenocarcinoma de células basales; MC: Carcinoma mucoepidermoide; SCC: Carcinoma de células escamosas; MC: Carcinoma mioepitelial; LC: Carcinoma linfoepitelial; PLGA: Adenocarcinoma polimorfo de bajo grado.

a los criterios de clasificación de los tumores híbridos. Toshitaka y cols desarrollan este dilema compartido por otros autores, indicando que en los casos donde los tipos histológicos provinieran de caminos de diferenciación comunes, se clasificarían como tumores no híbridos pero en los casos donde, a pesar de esto, cada carcinoma ocupara más del $30 \%$ del tumor o el grado de proliferación celular de cada tipo fuese distinto, debía considerarse como híbrido ${ }^{13}$.
La importancia de diferenciar la histología y el grado de malignidad en el caso de los tumores híbridos radica en el pronóstico y comportamiento distinto que puede tener cada tipo histológico $y$, en consecuencia, nos permite determinar la mejor terapia. Dado que existe poca información disponible acerca del pronóstico de dichos tumores, diversos autores concuerdan que el componente con mayor grado de malignidad determinaría el comportamiento bio- 
lógico de la neoplasia, su pronóstico y manejo ${ }^{13,15,18-22}$.

\section{CONCLUSIÓN}

La parótida corresponde a la localización de mayor frecuencia de los tumores de glándulas salivales y, dentro de ella, la mayoría son entidades benignas y se presentan como aumento de volumen. Nuestro caso correspondió a una neoplasia maligna de parótida y su presentación clínica fue atípica, con tumoración en conducto auditivo externo. Es por esto que la sospecha clínica y el estudio acabado de estas lesiones es muy importante. La mayoría de las neoplasias de parótida derivan de un único tipo histológico, pero eventualmente puede desarrollarse más de un tipo dentro de una misma glándula dando lugar a distintas entidades, dentro de ellos, los tumores híbridos de glándulas salivales. Este trabajo corresponde al segundo reporte de la literatura de un tumor parotídeo compuesto por carcinoma adenoideo quístico y carcinoma basaloideo. Es muy relevante la identificación de la histología y grado de malignidad de estos tipo de tumores, ya que determinan el pronóstico y tratamiento de ellos.

\section{BIBLIOGRAFÍA}

1. ANSARI MH. Salivary gland tumors in an Iranian population: A retrospective study of 130 cases. J Oral Maxillofac Surg 2007; 65: 2187-94.

2. Aparecida de Oliveira F, Barroso E, Teixeira C, Abreu A, Carvalho de Aquino E, Cassia R, Franco E. Salivary Gland Tumor: A Review of 599 Cases in a Brazilian Population. Head and Neck Pathol 2009; 3: 271-5.

3. Barnes L, Eveson JW, Reichart P, Sidransky D. Pathology and Genetics of Head and Neck Tumours. World Health Organization Classification of Tumors, IARC Press, Lyon. 2005.

4. Whitt J, Schafer D, Callihan M. Multiple Malignant Salivary Gland Neoplasms: Mucoepidermoid Carcinoma of Palate and Adenoid Cystic Carcinoma of Floor of Mouth. Head and Neck Pathol 2008; 2: 41-8.
5. Jones AV, Craig GT, Speight PM, Franklin CD. The range and demographics of salivary gland tumours diagnosed in a UK population. Oral Oncol 2008; 44: 407.

6. Speight PM, Barrett AW. Salivary gland tumours. Oral Dis 2002; 8: 229.

7. SPIRo RH. Salivary neoplasms: overview of a 35year experience with 2,807 patients. Head Neck Surg 1986; 8: 177.

8. Guzzo M, Locati LD, Prott FJ, Gatta G, McGurk M, LICITRA L. Major and minor salivary gland tumors. Critical Reviews in Oncology/ Hematology 2010; 74: 134-48.

9. Licitra L, Grandi C, Prott FJ, et al. Major and minor salivary glands tumours. Crit Rev Oncol Hematol 2003; 45: 215.

10. Celedón C, Ojeda JP, Agurto M, Olavarría C, Paredes A, Niklischek E. Tumores de glándulas salivales. Experiencia de 20 años. Rev Otorrinolaringol Cir Cab-Cuello 2002; 62: 25564.

11. Seifert G, Donath K. Multiple tumors of the salivary glands :terminology, nomenclature. Eur J Cancer B Oral Oncol 1996; 32B: 3.

12. Gnepp DR, Schroeder W, Heffner D. Synchronous tumors arising in a single major salivary gland. Cancer 1989; 63: 1219-24.

13. Toshitaka N, Isamu S, Yasuo I, Akira A. Hybrid carcinomas of the salivary glands: Report of nine cases with a clinicopathologic, Inmunohistochemical, and p53 Gene Alteration Analysis. Mod Pathol 2002; 15(7): 724-33.

14. Oliva J, Ramírez-Velásquez J, Zaldívar F, Hurtado L. Diagnóstico de neoplasias en glándula parótida: Características clínicas y biopsia por aspiración con aguja fina. Cirujano General2005; 27: 18-21.

15. Selfert G, Donath K. Hybrid tumours of salivary glands. Definition and classification of five rare cases. Eur J Cancer B Oral Oncol 1996; 32B(4): 251-9.

16. Nagao T, Sugano I, Ishida Y, Asoh A, Munakata $S$, Yamazaki K, Konno A, Imaya K, Shimizu T, Serizawa $\mathrm{H}$, EBIHARA Y. Hybrid carcinomas of the salivary glands: report of nine cases with a clinicopathologic, immunohistochemical, and p53 gene alteration analysis. Mod Pathol 2002; 15(7): 724-33. 
17. Ellis GL, Auclair PL, Gnepp DR. Surgical pathology of the salivary glands. WB Saunders, Philadelphia. 455-88, 1991.

18. Ruíz-Godoy LM, Mosqueda-Taylor A, SuárezRoa L, Poitevin A, Bandala-Sánchez E, Meneses-García A. Hybrid tumours of the salivary glands. A report of two cases involving the palate and a review of the literature. Eur Arch Otorhinolaryngol 2003; 260(6): 3.

19. Kamio N, Tanaka Y, Mukal M, Ikeda E, Kuramochi S, FuJII M ET AL. A hybrid carcinoma, adenoid cystic carcinoma and salivary duct carcinoma of the salivary gland: an immunohistochemical study. Virchows Arch 1997; 430: 495-500.

20. Snyder ML, Paulino AF. Hybrid carcinoma of the salivary gland: salivary duct adenocarcinoma and adenoid cystic carcinoma. Histopathology 1999; 35: 380-3.

21. Croitoru CM, Suarez PA, Luna MA. Hybrid carcinomas of salivary glands. Report of 4 cases and review of the literature. Arch Pathol Lab Med 1999; 123: 698-702.

22. Chetty R, Medley P, Essa A. Hybrid carcinoma of salivary glands. Arch Pathol Lab Med 2000; 124 : 494-6. 